\title{
TEV GAMMA RAY EMISSION FROM THE ACTIVE GALACTIC
} NUCLEUS MARKARIAN 421

\section{The Whipple Collaboration}

M. Schubnell, ${ }^{1}$ C. W. Akerlof, ${ }^{1}$ M. F. Cawley, ${ }^{2}$ M. Chantell,${ }^{3}$ V. Connaughton,${ }^{3}$ D. J. Fegan,${ }^{4}$ S. Fennell, ${ }^{3}$ J. A. Gaidos,${ }^{5}$ A. M. Hillas, ${ }^{6}$ A. D. Kerrick, ${ }^{7}$ P. Kwok, ${ }^{7}$ R. C. Lamb, ${ }^{7}$ D. A. Lewis, ${ }^{7}$ D. I. Meyer,${ }^{1}$ G. Mohanty, ${ }^{7}$ J. Rose, ${ }^{6}$ A. C. Rovero, ${ }^{3}$ G. Sembroski,${ }^{5}$ T. C. Weekes, ${ }^{3}$ M. West,${ }^{3}$ T. Whitaker,${ }^{3}$ and C. Wilson ${ }^{5}$

${ }^{1}$ University of Michigan, Ann Arbor, MI 48109 USA

${ }^{2}$ St. Patrick's College, Maynooth, Co. Kildare, Ireland

3 Whipple Observatory, Harvard-Smithsonian CfA, Amado, AZ 85645 USA

${ }^{4}$ University College Dublin, Belfield, Dublin 4, Ireland

` Purdue University, West Lafayette, IN 47907 USA

${ }^{6}$ University of Leeds, Leeds LS2 9JT, UK

${ }^{7}$ Iowa State University, Ames, IA 50011 USA

\begin{abstract}
$\mathrm{TeV}$ energy gamma ray emission from the blazar Markarian 421 was detected with the Whipple 10 meter imaging Cherenkov telescope initially during the observation period March-June 1992 at an average photon flux of $1.5 \times 10^{-11} \mathrm{~cm}^{-2} \mathrm{~s}^{-1}$. The photon spectrum in the $\mathrm{TeV}$ range lies on the extrapolation of the data points observed by EGRET during viewing phase I and indicates that the emission follow's an unchanged power law between $100 \mathrm{MeV}$ and $10 \mathrm{TeV}$ with no significant intergalactic absorption. More recent observations from the spring of 1993 confirm the 1992 measurements. Here we give an update on the status of the observations, the analysis for time variability, and the results from the analysis to determine the spectral index for Markarian 421 at $\mathrm{TeV}$ energies.

The search for $\mathrm{TeV}$ emission from other extragalactic sources detected by the EGRET instrument on the Compton Gamma Ray Observatory at $\mathrm{GeV}$ energies have so far been negative.
\end{abstract}

\section{INTRODUCTION}

The BL Lac object Markarian 421 belongs to a particular class of Active Galactic Nuclei characterized by compact radio emission, strong optical polarization and rapid variability at optical, IR and $\mathrm{x}$-ray frequencies. The pattern of variability for MRK 421 is erratic and variations on time scales of days (optical) and hours (x-ray) are reported.

Active Galactic Nuclei are, because of their extremely high non-thermal luminosity, favoured candidates as the main source for the cosmic ray flux. Additional evidence for this picture came with the detection of $\mathrm{GeV}$ gamma ray emission from 
some 20 AGN by the EGRET detector aboard the Compton Gamma Ray Observatory (Fichtel et al., 1993) and the subsequent observation of $\mathrm{TeV}$ photons from MRK 421 (Punch et al., 1992). While some of the AGNs observed by EGRET show strong flux variability at $\mathrm{GeV}$ energies, no evidence for time variability was reported from Markarian 421 (Lin et al., 1993).

Flux variations are of strong interest because the time scale of variability is closely related to the size of the emission region. In a widely accepted picture, it is assumed that most of the emission originates from the central engine of a blazar. The short time scale variations indicate the existence of a very compact object, presumably a supermassive black hole, with $M>10^{6} M_{\odot}$ which is fed by accretion. The observed gamma rays at $\mathrm{GeV} \cdot \mathrm{TeV}$ energies are more likely to be produced in the jet region since the compact inner source is opaque to high energy gamma rays through pair production.

\section{EXPERIMENT and Shower IMAge SELEction}

The observations reported in this paper were carried out with the very high energy $\gamma$-ray telescope (Cawley et al., 1990) at the Whipple Observatory on Mt. Hopkins in southern Arizona ( $31^{\circ} 41^{\prime}$ north, $110^{\circ} 53^{\prime}$ west, alt $=2300 \mathrm{~m}$ ). The 10 meter diameter telescope images Cherenkov light from air showers on a hexagonal array of 109 fast photomultiplier tubes in the focal plane. The inner 91 oneinch tubes of this high resolution camera have a $0.25^{\circ}$ full field of view and are surrounded by an outer ring of 18 two-inch tubes. The total field of view for the camera is $3.5^{\circ}$. A shower image is recorded when at least $m$ out of the inner 91 tubes exceed the threshold of $n$ photoelectrons. With the standard trigger condition $(m=2, n=40)$ a typical trigger rate of $3 \mathrm{~Hz}$ is obtained.

The vast majority of atmospheric air showers are produced by charged atomic nuclei and have to be discriminated against those which are originated by gamma rays. The most sensitive technique yet employed by the Whipple Collaboration for this purpose, 'supercuts', (Reynolds et al., 1993) uses four parameters to characterize the approximately elliptical shower image. Monte Carlo simulations (Hillas 1985, Macomb 1990) and repeated observations of the Crab Nebula (Weekes et al., 1989, Vacanti et al., 1991) demonstrate that the Cherenkov light images of air showers induced by $\gamma$-rays can be reliably distinguished from the hadronic background.

\section{ANALYSIS AND RESULTS}

In 1992 observations of MRK 421 were made between March 24 and June 2, for a total of 7.5 hours on-source in the standard on/off tracking mode (Reynolds et al., 1993). The observed excess has a significance of 6.3 sigma and corresponds to an average photon flux of $1.5 \times 10^{-11} \mathrm{~cm}^{-2} \mathrm{~s}^{-1}$ above $0.5 \mathrm{TeV}$, equivalent to 0.3 that of the intensity of the Crab Nebula (Punch et al., 1992). 
During the last winter, the AGN observation program was continued and between December 23, 1992 and March 20, 1993, Markarian 421 was observed on 11 clear nights for a total of 806 minutes. However, in the fall of 1992, several instrumental improvements were made including the mounting of light cones in front of the photomultiplier tubes and the recoating of most the mirrors. The preparation for stereo operation with the nearby new 11 meter telescope (Schubnell et al., 1992) led us also to incorporate a two-level trigger and a change to a lower threshold at higher trigger multiplicity $(m=3, n=30)$. These modifications had the effect of changing the optimum selection criteria used for selecting the gamma-ray images; since these have not yet been optimized on the Crab Nebula signal we have chosen to use the 'supercuts' selection on all the data with a trigger introduced in software to make the energy thresholds similar to that used previously. We also will quote all flux limits for the 1993 observing period in terms of equivalent 'Crab' flux units (where we have analyzed contemporaneous observations of the Crab Nebula in the same way). The average photon flux from MRK 421 is equivalent to $0.3 \mathrm{Crab}$ flux units during the observing period winter 1992 and 0.28 Crab flux units during winter 1993.

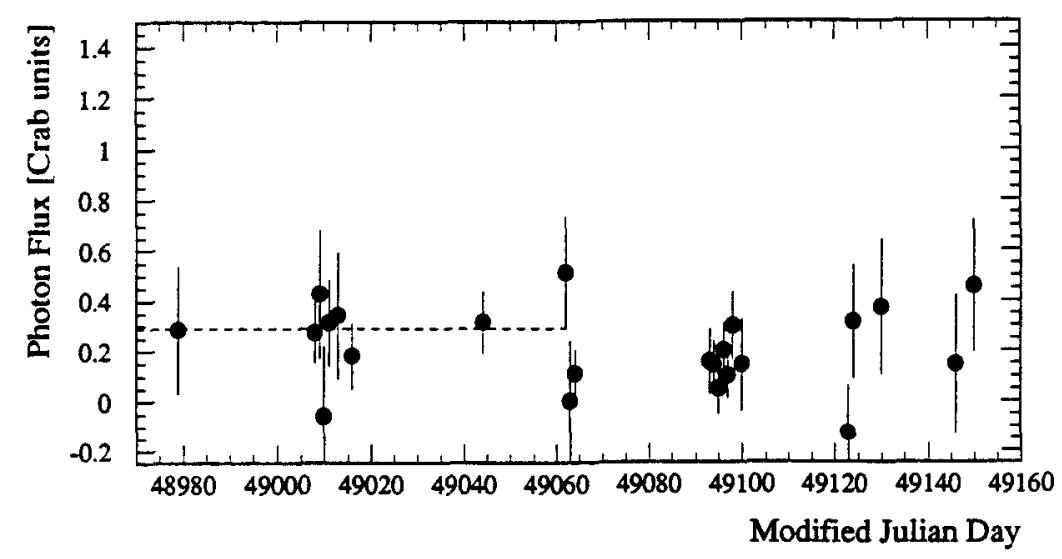

Figure 1. The observed fluxes from Markarian 421 for the viewing period winter 1993. Each data point corresponds to one night of observation. All errors are statistical and vary with the observing duration. The dashed line indicates the average observed photon flux during the time of contemporaneous CRAB observations,

In an effort to search for variability on an shorter time scale, we divided the data into daily intervals. This is shown in Fig. I for the 1993 data set. The maximum deviation (3.3 $\sigma$ ) from a steady flux is found on MJD 48764. A $\chi^{2}$ test of the 1992 data gives a $4.5 \times 10^{-2}$ probability that the observed flux is consistent with being constant. For the first half of the 1993 viewing period, the period were we have contemporaneous $\mathrm{CRAB}$ observations, we find the probability for 
constant emission to be $8.0 \times 10^{-1}$. For the later observations we can only give an estimate for the flux based on the non-contemporaneous CRAB observations (see Fig.1).

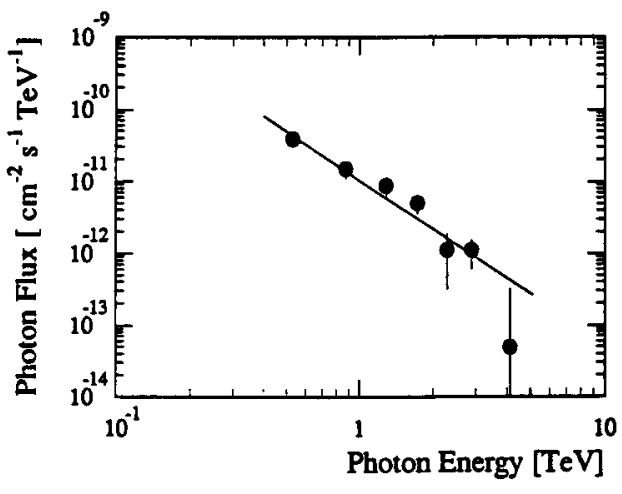

Figure 2. The differential energy spectrum of Markarian 421 from observations during 1992 (Mohanty et al., 1993).

For the determination of the energy spectra the standard 'supercut' selection procedure is not ideal. A bias against higher energy gamma ray showers makes it necessary to extend the method and to include Monte Carlo simulations in order to extract an undistorted spectrum (Lewis et al., 1993). When applying this procedure to our data taken in 1992 we obtain as best power-law fit for the differential spectrum:

$$
d N / d E=1.04( \pm 0.14) \times 10^{-11} E^{-2.25 \pm 0.19 \pm 0.3} \text { photonscm }{ }^{-2} s^{-1} \mathrm{TeV}^{-1}
$$

Fig. 2 shows the fit along with the data points from the 1992 data set. Up to the highest observed energies we see no break in the spectrum and hence no evidence for absorption on intergalactic infrared photons. This leads to a constraint on the intergalactic infrared energy density (Stecker and De Jager, 1993).

The launch of the Compton Gamma Ray Observatory and the subsequent detection of more than 20 extragalactic objects as powerful $\mathrm{GeV}$ gamma ray emitters by the EGRET experiment provided us with a catalog of potential $\mathrm{TeV}$ gamma ray sources. Since winter 1991 we assigned a high priority to the observation of AGN. The results of our extragalactic source search are summarized in Tab. 1. So far we find no evidence for $\mathrm{TeV}$ emission from any AGN other than Markarian 421. The upper limits quoted in the table are calculated at the $3 \sigma$ level. In view of the reported flux variability of most of these objects it is important to emphasize that these limits pertain only for the limited epoch of the observations.

\section{ACKNOWLEDGEMENTS}

This research was supported by the US Department of Energy, NASA, the Smithsonian Scholarly Studies Research Fund, and EOLAS, the scientific funding agency of Ireland. 


\section{Schubnell et al. 601}

\begin{tabular}{|c|c|c|c|c|c|c|}
\hline $\begin{array}{l}\text { Coordinate } \\
\text { designation }\end{array}$ & Name & Type & $\mathrm{z}$ & $\begin{array}{l}\text { Flux } \\
\left(10^{-11} \mathrm{~cm}^{-2} \mathrm{~s}^{-1}\right)\end{array}$ & $\begin{array}{l}E_{t h} \\
{[\mathrm{TeV}]}\end{array}$ & $\begin{array}{c}\text { EGRET } \\
\text { source }\end{array}$ \\
\hline $0116+319$ & $4 \mathrm{C} 31.04$ & Gal. Pair & 0.059 & $<1.1$ & 0.4 & \\
\hline $0232-090$ & MKN 1048 & Seyfert & 0.043 & $<2.2$ & 0.5 & \\
\hline $0241+622$ & $4 \mathrm{U} 241$ & Seyfert & 0.044 & $<4.3$ & 0.4 & \\
\hline $0316+413$ & NGC 1275 & Seyfert & 0.018 & $<1.5$ & 0.4 & \\
\hline $0415+375$ & $3 \mathrm{C} 111$ & Seyfert & 0.044 & $<2.0$ & 0.4 & \\
\hline $0528+134$ & PKS & OVV & 1.9 & $<3.0$ & 0.5 & * \\
\hline $0716+714$ & S5 & BL Lac & $\ldots$ & $<2.8$ & 0.5 & * \\
\hline \multirow[t]{2}{*}{$0836+710$} & $4 C+71.07$ & QSO & 2.17 & $<4.7$ & 0.4 & * \\
\hline & & & & $<2.3$ & 0.6 & \\
\hline $0851+202$ & OJ 287 & BL Lac & 0.306 & $<2.7$ & 0.4 & \\
\hline $1101+384$ & MKN 421 & BL Lac & 0.031 & 1.5 & 0.5 & * \\
\hline $1208+396$ & NGC 4151 & Seyfert & 0.003 & $<2.3$ & 0.4 & \\
\hline $1215+303$ & ON 325 & BL Lac & 0.24 & $<2.9$ & 0.4 & \\
\hline $1219+285$ & W Com & BL Lac & 0.102 & $<2.2$ & 0.4 & \\
\hline \multirow[t]{2}{*}{$1226+023$} & $3 \mathrm{C} 273$ & QSO & 0.158 & $<4.8$ & 0.4 & * \\
\hline & & & & $<2.0$ & 0.55 & \\
\hline $1253-055$ & 3 C 279 & QSO & 0.538 & $<0.8$ & 0.6 & $\star$ \\
\hline $1633+382$ & $4 C \quad 38.41$ & ovV & 1.814 & $<0.5$ & 0.5 & * \\
\hline $1652+398$ & MKN 501 & BL Lac & 0.034 & $<0.7$ & 0.5 & \\
\hline $1727+502$ & I $\mathrm{ZW} \quad 187$ & BL Lac & 0.055 & $<0.4$ & 0.5 & \\
\hline $2200+420$ & BL LAC & BL Lac & 0.069 & $<0.7$ & 0.5 & \\
\hline $2251+158$ & $3 \mathrm{C} \quad 454.3$ & Seyfert & 0.859 & $<1.2$ & 0.45 & $\star$ \\
\hline $2304+042$ & PG2304 & Seyfert & 0.042 & $<1.9$ & 0.5 & \\
\hline
\end{tabular}

Table 1. List of extragalactic objects observed with the Whipple $10 \mathrm{~m}$ telescope in 1992 and 1993. The flux limits quoted in column 5 are 3 sigma limits above the energy threshold $E_{t h}$. The $\star$ in column 6 indicates that this source was detected by EGRET.

\section{REFERENCES}

M. F. Cawley et al., Exper. Astr. 1 (1990) 173.

C. E. Fichtel et al., Proc. Compton Observatory Symp. (St. Louis) (1992).

A. M. Hillas, in Proc. 19th ICRC (La Jolla) 3 (1985) 445.

Y. C. Lin et al., Ap. J. (1993) L61

D. A. Lewis et al., in Proc. 23st ICRC (Calgary) 1 (1993) 279.

D. J. Macomb \& R. C. Lamb, in Proc. 21st ICRC (Adelaide) 2 (1990) 435.

G. Mohanty et al., in Proc. 23st ICRC (Calgary) 1 (1993) 440.

M. Punch et al., Nature 358 (1992) 477.

P. T. Reynolds et al., Ap. J. 404 (1993) 467.

M. Schubnell et al., Proc. Compton Observatory Symp. (St. Louis) (1992).

F. W. Stecker and O. C. De Jager, Ap. J. (1993), in press.

G. Vacanti et al., Ap. J. 377 (1991) 467.

T. C. Weekes et al., Ap. J. 342 (1989) 379. 